\title{
Efeito da densidade de estocagem sobre o biofilme e o desempenho de pós-larvas do camarão-rosa Farfantepenaeus paulensis cultivadas em gaiolas
}

\author{
Effect of stocking density on the biofilm and the performance of post-larvae of the \\ pink shrimp Farfantepenaeus paulensis in cages
}

\author{
Artur de Lima Preto ${ }^{1}$ Ronaldo Cavalli ${ }^{2}$ Tito Pissetti $^{3}$ \\ Paulo César Abreu ${ }^{4}$ Wilson Wasielesky Júnior ${ }^{5}$
}

RESUMO

O objetivo deste estudo foi analisar a influência da densidade de estocagem na sobrevivência e no crescimento de pós-larvas de $\boldsymbol{F}$. paulensis cultivadas em gaiolas durante a fase de berçário. Além disso, foram identificados $e$ quantificados os principais microrganismos presentes no biofilme formado nos substratos artificiais colocados dentro das gaiolas. Foram usadas gaiolas com $4 \mathrm{~m}^{2}$ de fundo e com substratos artificiais, nas densidades de 100, 200, 300, 400 e $500 P L_{25} \mathrm{~m}^{-2}$. O experimento durou 35 dias. Observou-se uma relação negativa entre o aumento da densidade de estocagem de camarões e a sobrevivência e o crescimento destes. Foi observada uma preferência pelo consumo de diatomáceas cêntricas. A viabilidade técnica do cultivo de $\boldsymbol{F}$. paulensis em gaiolas foi confirmada. O cultivo de $\boldsymbol{F}$. paulensis em gaiolas na fase de berçário pode ser feito em densidades de até 400PL $\mathrm{m}^{-2}$, sem maiores perdas em sua sobrevivência e seu crescimento.

Palavras-chave: aqüicultura; camarões; berçário; gaiolas; biofilme; microalgas; diatomáceas.

\section{ABSTRACT}

The aim of this study was to analyze the influence of the stocking density in the survival and growth of Farfantepenaeus paulensis postlarvae reared in cages during the nursery phase. Additionally, we identified and quantified the main microorganisms present on the biofilm formed on artificial substrates placed inside the cages. We used $4 \mathrm{~m}^{2}$ bottom surface cages with artificial substrates and shrimp stocking densities of 100, 200, 300, 400 and 500PL $\mathrm{P}_{25} \mathrm{~m}^{-2}$. The experiment lasted for 35 days. A negative relationship was observed between the increase of shrimp stocking density and their survival/growth rates. A preference for centric diatoms was also observed. The technical feasibility of culturing $\mathbf{F}$. paulensis in cages was confirmed. The rearing of $\boldsymbol{F}$. paulensis in nursery cages may be conduced in densities at also 400PL $\mathrm{m}^{-2}$, without more loss in survival and growth.

Key words: aquaculture; shrimps; nursery; cages; biofilm; microalgae; diatoms.

\section{INTRODUÇÃO}

A aqüicultura, definida como o "cultivo de organismos aquáticos, incluindo peixes, moluscos, crustáceos, anfíbios e plantas aquáticas" (BARDACH et al., 1972), é atualmente a atividade de maior crescimento entre os setores de produção animal (FAO, 2002). Dentre os produtos oriundos da aqüicultura, o camarão marinho apresenta um alto valor econômico. Os países asiáticos são os principais produtores

${ }^{1}$ Programa de Pós-graduação em Aqüicultura da Fundação Universidade Federal do Rio Grande (FURG), Rio Grande, RS, Brasil. Email: arturlp@uol.com.br

${ }_{2}^{2}$ Fundação Universidade Federal do Rio Grande (FURG), Departamento de Oceanografia, Estação Marinha de Aquacultura, CP 474, 96201-900, Rio Grande, RS, Brasil. E-mail: cavalli@mikrus.com.br

${ }^{3}$ Programa de Pós-graduação em Aqüicultura, FURG, Rio Grande, RS, Brasil. E-mail: titolp@bol.com.br

${ }^{4}$ Departamento de Oceanografia, Laboratório de Ecologia do Fitoplâncton e de Microrganismos Marinhos, FURG, CP 474, 96201900, Rio Grande, RS, Brasil. E-mail: docpca@furg.br

${ }^{5}$ Departamento de Oceanografia, Estação Marinha de Aquacultura, FURG, CP 474, 96201-900, Rio Grande, RS, Brasil. E-mail: manow@mikrus.com.br 
mundiais deste crustáceo e o Brasil, embora seja apenas o sexto colocado ao nível mundial, é atualmente o maior produtor no hemisfério ocidental, com 90.000 toneladas produzidas em 2003 (ROCHA et al., 2004).

O cultivo de camarão é normalmente realizado em viveiros com custos relativamente elevados. SOUZA FILHO et al. (2002) estimaram que o custo de implantação de um hectare para o cultivo de camarão em Santa Catarina seria de aproximadamente $\mathrm{R} \$ 29.000,00$. Desta forma, comunidades de pescadores artesanais ficam praticamente impossibilitadas de cultivar camarão em viveiros. Por outro lado, em alguns países da Ásia o camarão é cultivado em gaiolas (tanques-rede) colocadas em ambientes naturais (WALFORD \& LAM, 1987; SINGH \& MATTAI, 1990; SAMARANAYAKE, 1990). Além do menor custo de implantação, o cultivo em gaiolas permite a utilização de áreas costeiras nas quais não é possível a construção de viveiros e se aproveita melhor a produtividade natural do ambiente.

No Brasil, vários autores têm estudado o cultivo de camarões em gaiolas. Apesar dos ótimos resultados obtidos com o camarão branco Litopenaeus vannamei (PAQUOTTE et al., 1998; OSTRENSKY \& PILCHOWSKY, 2002), o cultivo desta espécie, por ser exótica, enfrenta limitações legais (IBAMA, 1998) em vista do impacto potencial ao ambiente no caso de escape. Por outro lado, o camarão-rosa Farfantepenaeus paulensis, além de também ter apresentado resultados satisfatórios quando cultivado em gaiolas (WASIELESKY et al., 1995; BALLESTER et al., 2003; CAVALLI \& WASIELESKY, 2003; LOMBARDI et al., 2003; JENSEN et al., 2004), não apresenta as mesmas limitações legais, uma vez que é endêmica na costa sudeste e sul do Brasil (D'INCAO, 1995),

Associado ao cultivo de camarões em gaiolas, o desenvolvimento de estratégias para aumentar a produção primária pode resultar na melhoria da sobrevivência e no crescimento dos camarões, na qualidade da água e, eventualmente, reduzir os custos de produção. ABREU et al. (1998) relatam que microrganismos (bactérias, ciliados, flagelados e microalgas) presentes no biofilme aderido a substratos submersos podem representar uma fonte substancial de alimento para larvas e juvenis de $\boldsymbol{F}$. paulensis. A presença de biofilme aderido à parede dos tanques de cultivo resultou na melhoria da qualidade de água e no maior crescimento de $\boldsymbol{F}$. paulensis (THOMPSON et al., 2002). Neste mesmo trabalho, a análise do conteúdo estomacal de $\boldsymbol{F}$. paulensis indicou o consumo não seletivo do biofilme. BALLESTER et al. (2003) observaram melhores taxas de sobrevivência e crescimento de $\boldsymbol{F}$. paulensis com a adição de substratos em um cultivo em gaiolas. Estudos com outras espécies de camarões peneídeos também demonstraram a importância do biofilme na alimentação dos camarões (STONER \& ZIMMERMAM, 1988; MOSS \& PRUDER, 1995).

No cultivo de camarões, o berçário é uma fase intermediária entre a produção de pós-larvas em laboratório e a engorda no campo, caracterizando-se pelo uso de altas taxas de renovação de água, elevadas densidades de estocagem e fornecimento de alimentos inertes (SPECK et al., 1993). As vantagens da adoção do berçário incluem um melhor controle da produção, aumento na eficiência e previsibilidade do sistema de cultivo através da regulação do fluxo de pós-larvas para o povoamento das unidades de engorda, uma melhor avaliação dos estoques adquiridos, um maior controle sobre patógenos e alimentação e a homogeneização das características zootécnicas dos camarões (NUNES, 2002; SEIFFERT et al., 2003). Alguns autores (STURMER et al., 1992; RODRIGUEZ et al., 1993; SEIFFERT et al., 2003) afirmam também que o uso de berçários proporciona camarões maiores e mais tolerantes às condições ambientais, os quais geralmente atingem maiores taxas de sobrevivência e tamanho num menor período de cultivo.

Embora o cultivo de camarões em gaiolas apresente algumas vantagens em relação ao cultivo em viveiros, ainda não foram desenvolvidos estudos que definam um manejo adequado a esta forma de produção. Assim sendo, o presente artigo analisou a influência da densidade de estocagem na sobrevivência e no crescimento de $\boldsymbol{F}$. paulensis na fase de berçário em gaiolas. Além disso, a composição dos microrganismos no biofilme formado nos substratos artificiais colocados dentro das gaiolas foi identificada e quantificada com o intuito de se verificar se existe alguma preferência alimentar do camarão $\boldsymbol{F}$. paulensis.

\section{MATERIAL E MÉTODOS}

O experimento foi realizado na enseada do Saco do Justino, estuário da Lagoa dos Patos (Rio Grande, RS), com início em 22/12/2003 e com uma duração de 35 dias. As pós-larvas (PL) de camarãorosa $\boldsymbol{F}$. paulensis foram produzidas na Estação Marinha de Aquacultura (EMA) de acordo com MARCHIORI (1996).

Pós-larvas com 25 dias $(0,014 \mathrm{~g} \pm 0,013)$ foram cultivadas em gaiolas de poliéster revestido com PVC $\operatorname{com} 4 \mathrm{~m}^{2}$ de fundo e malha com abertura de $1,5 \mathrm{~mm}$, em cinco diferentes densidades de estocagem $(100,200$, 
300, 400 e 500PL m ${ }^{-2}$ ) com três repetições cada. As gaiolas foram montadas em linha, com uma distância média de $30 \mathrm{~cm}$ entre si e com seus fundos distantes em média $20 \mathrm{~cm}$ do fundo da lagoa. As gaiolas foram colocadas no ambiente 20 dias antes do povoamento para que ocorresse a formação do biofilme, conforme proposto por SANTOS (2003). Nesse mesmo dia, substratos artificiais (três telas brancas de polietileno de 1,9 x $0,7 \mathrm{~m}$ e malha com abertura de $1 \mathrm{~mm}$ ) foram colocados em cada gaiola de forma a aumentar em $100 \%$ a superfície interna lateral. Os camarões foram alimentados ad libitum em quatro refeições diárias com uma ração comercial específica para a fase de berçário de camarões marinhos com $40 \%$ de proteína bruta (Camaronina, Purina). A transparência (Disco de Secchi), temperatura (termômetro de mercúrio com precisão de $0,5^{\circ} \mathrm{C}$ ) e salinidade da água (refratômetro ótico com precisão $1 \%$ ) foram medidas diariamente às $8: 00 \mathrm{~h}$, sendo a temperatura medida novamente às 18:00h. A concentração de oxigênio dissolvido (oxímetro Handylab OXI/Set SCHOTT), o pH (pHmetro Handylab 2 BNC SCHOTT) e a amônia total (UNESCO, 1983) foram medidas em cada gaiola e em um ponto controle distante 100 metros do local de cultivo a cada 14 dias às 8:00h e às 18:00h, com exceção da amônia total, medida apenas às 18:00h. Para se analisar possíveis relações entre a velocidade do vento e as concentrações de oxigênio dissolvido na água durante a realização do estudo, foram utilizados os dados de velocidade de vento coletados a cada hora pela Estação Meteorológica da FURG em Rio Grande, RS.

A cada 14 dias, foi feita a amostragem de dois pedaços de $2 \times 2 \mathrm{~cm}$ do substrato artificial de cada gaiola coletados a $20 \mathrm{~cm}$ da superfície da água para que fosse feita a determinação das concentrações de clorofila $a$ no biofilme formado nos substratos artificiais, assim como a identificação e quantificação dos organismos presentes nestes. A extração do pigmento fotossintético, a determinação da concentração de clorofila $a$, bem como a identificação e quantificação dos organismos presentes no biofilme formado nos substratos, foram feitos de acordo com BALLESTER et al. (2003). Ao final do experimento, 30 camarões de cada gaiola foram pesados e os demais foram contados individualmente.

Os dados obtidos neste estudo foram submetidos ao teste de Lévene e ao teste K-S para analisar se os mesmos cumpriam os pré-requisitos da análise de variância (ANOVA). Os dados de peso final dos camarões, como não cumpriam os pré-requisitos, foram transformados pela função $f=1 /(\mathrm{x}+1)$. Alguns dados de densidade de microrganismos obtidos 14 dias após o início do estudo (densidade de diatomáceas cêntricas) também não os cumpriam, sendo transformados com a função $f=\sqrt{ }(x+0,5)$. Feitas as transformações necessárias, aplicou-se a ANOVA univariada (á $=0,05)$ e, ocorrendo diferencas significativas, foi aplicado o teste de Tukey. Alguns dados de densidade de microrganismos obtidos 28 dias após o início do estudo (densidade de diatomáceas penadas $=50 \mu \mathrm{m}$ de comprimento) não cumpriam os pré-requisitos da ANOVA, nem quando tinham seus valores transformados, e foram, portanto, analisados com o teste não-paramétrico de KruskalWallis $(\mathrm{a}=0,05)$.

\section{RESULTADOS E DISCUSSÃO}

Os valores dos parâmetros físico-químicos da água (Tabela 1) não apresentaram diferenças significativas entre os tratamentos $(p>0,05)$. Entretanto, ocorreram diferenças significativas nas concentrações médias de oxigênio dissolvido e $\mathrm{pH}$ entre o ponto controle $\left(5,97 \mathrm{mg}^{-1}\right.$ e 8,58 , respectivamente) e as gaiolas experimentais $\left(4,24 \mathrm{mg} \mathrm{l}^{-1}\right.$ e 8,31, respectivamente). A baixa salinidade durante o período experimental (média de 5,9\%o) pode ter limitado o crescimento dos camarões, mas provavelmente não influenciou de forma significativa a sobrevivência dos animais (CORLETO et al., 1993; WASIELESKY, 2000; TSUZUKI et al., 2003). A temperatura da água no mesmo período (mínima de $21^{\circ} \mathrm{C}$ e máxima de $31^{\circ} \mathrm{C}$ ) sempre esteve dentro da faixa considerada aceitável para a espécie (SOARES, 1996; HENNIG \& ANDREATTA, 1998), assim como o $\mathrm{pH}$ (mínimo de 7,83 e máximo de 8,88) (BOYD, 1990) e a concentração média de amônia total (mínima de $0,00 \mathrm{mg} \mathrm{l}^{-1}$ e máxima de $0,12 \mathrm{mg} \mathrm{l}^{-1}$ ) (OSTRENSKY \& WASIELESKY, 1995; WASIELESKY,

Tabela 1 - Valores médios ( \pm DP), mínimos e máximos de salinidade, temperatura $\left({ }^{\circ} \mathrm{C}\right)$ às 8:00 e 18:00 horas, transparência $(\mathrm{cm})$, concentração de oxigênio dissolvido $(\mathrm{mg} / \mathrm{l})$ nas gaiolas e no ponto controle, $\mathrm{pH}$ nas gaiolas e no ponto controle e concentração de amônia total (mg/l) nas gaiolas e no ponto controle.

\begin{tabular}{llll}
\hline & Média \pm DP & Mínimo & Máximo \\
\hline Salinidade & $5,9 \pm 1,8$ & 3,0 & 10,0 \\
Temperatura - 8:00 & $23,8 \pm 1,6$ & 21,0 & 27,0 \\
Temperatura - 18:00 & $27,2 \pm 2,1$ & 24,0 & 31,0 \\
Transparência & $86 \pm 16$ & 50 & 120 \\
OD - Gaiolas & $4,24 \pm 2,73$ & 0,37 & 7,86 \\
OD - Controle & $5,97 \pm 1,41$ & 4,45 & 8,80 \\
pH - Gaiolas & $8,31 \pm 0,32$ & 7,83 & 8,88 \\
pH - Controle & $8,58 \pm 0,35$ & 8,00 & 9,00 \\
Amônia Total - Gaiolas & $0,06 \pm 0,04$ & 0,00 & 0,12 \\
Amônia Total - Controle & $0,04 \pm 0,04$ & 0,00 & 0,08 \\
\hline
\end{tabular}


2000). Apesar da concentração média de oxigênio dissolvido nas gaiolas ter sido $4,24 \mathrm{mg} \mathrm{l}^{-1}$, valores inferiores a $1 \mathrm{mg} \mathrm{l}^{-1}$ foram observados, valores estes que possivelmente influenciaram negativamente a taxa de sobrevivência dos camarões (BOYD, 1990; POERSCH \& MARCHIORI, 1992). Embora em algumas ocasiões, as concentrações de oxigênio dissolvido no ponto controle tenham diminuído, estas quedas foram menores do que as observadas nas gaiolas. Isso provavelmente ocorreu devido à baixa circulação de água no interior das gaiolas, que foram confeccionadas com malha de pequena abertura, dificultando a passagem da água, principalmente se considerarmos que o biofilme formado nas malhas pode ter diminuído ainda mais a circulação. A velocidade média do vento durante o período experimental foi de $21 \mathrm{~km} \mathrm{~h}^{-1}$. Entretanto, nos dias em que se constataram baixas concentrações de oxigênio dissolvido na água, a velocidade do vento esteve muito abaixo da média, principalmente antes do amanhecer, o que torna provável a influência negativa da baixa velocidade do vento na concentração de oxigênio dissolvido dentro das gaiolas.

As concentrações de clorofila $a$ e as densidades das microalgas nos substratos artificiais são mostrados na tabela 2. As concentrações de clorofila $a$ nos substratos artificiais aumentaram no decorrer do estudo, não apresentando diferenças significativas entre os tratamentos. Os microrganismos presentes nos substratos foram classificados como diatomáceas cêntricas, diatomáceas penadas grandes $(\geq 50 \mu \mathrm{m}$ de comprimento) e diatomáceas penadas pequenas $(<50 \mu \mathrm{m}$ de comprimento). Com relação à densidade média de diatomáceas cêntricas, pôde-se observar que esta se manteve aparentemente constante no tratamento com 100PL m ${ }^{-2}$. No tratamento com 200PL $\mathrm{m}^{-2}$, a densidade das diatomáceas cêntricas teve um aparente aumento no $14^{\circ}$ dia de experimento, seguido de queda aos 28 dias. Isto diferiu dos tratamentos com 300 e 500PL m ${ }^{-2}$, no qual é observada uma queda de densidade seguida de um leve aumento. No tratamento com 400PL m ${ }^{-2}$, a densidade diminuiu com o decorrer do estudo. Apesar dessas variações, somente foram observadas diferenças significativas nas densidades de diatomáceas cêntricas entre os tratamentos no $14^{\circ}$ dia de estudo, quando foram observadas maiores densidades nos tratamentos com menor densidade de estocagem de camarões $(p<0,05)$. No caso das densidades de diatomáceas penadas, observou-se uma quantidade muito maior de diatomáceas penadas pequenas em relação às diatomáceas penadas grandes. Pôde-se também observar que, em quase todos os tratamentos, ocorreu um pequeno aumento na densidade de diatomáceas penadas grandes durante o experimento, ao contrário das diatomáceas penadas pequenas, onde, na maioria dos tratamentos, ocorre uma queda na densidade aos 14 dias de cultivo, seguida de um aumento aos 28 dias. Pôdese observar que aos 14 dias de cultivo a densidade de diatomáceas penadas grandes foi maior nos tratamentos de maior densidade de estocagem, sendo que estas diferenças não são mais observadas aos 28 dias. $\mathrm{O}$ contrário pôde ser visto em relação às densidades de diatomáceas penadas pequenas, nas quais aos 14 dias de cultivo, não foram observadas diferenças significativas, mas, aos 28 dias, uma densidade significativamente maior de diatomáceas foi observada no tratamento com 500PL m ${ }^{-2}$.

$\mathrm{O}$ aumento das concentrações médias de clorofila $a$ nos substratos artificiais, durante o cultivo $\left(8,18 \mu \mathrm{g} / \mathrm{cm}^{2}\right.$ ao final do período experimental), superou o valor máximo de $5,5 \mu \mathrm{g} \mathrm{cm}^{-2}$ observado por BALLESTER et al. (2003), embora as densidades finais

Tabela 2 - Valores médios ( \pm DP) de concentração de clorofila $a$ $\left(\mu \mathrm{g} . \mathrm{cm}^{-2}\right)$, densidade de diatomáceas cêntricas $\left(\mathrm{n}^{\mathrm{o}} \times 10^{5} . \mathrm{cm}^{-2}\right)$, densidade de diatomáceas penadas grandes (comprimento $=$ $50 \mu \mathrm{m})\left(\mathrm{n}^{\mathrm{o}} \times 10^{5} . \mathrm{cm}^{-2}\right)$ e densidade de diatomáceas penadas pequenas (comprimento $<50 \mu \mathrm{m}) \quad\left(\mathrm{n}^{\mathrm{o}} \times 10^{5} . \mathrm{cm}^{-2}\right)$ nos substratos artificiais do cultivo de Farfantepenaeus paulensis em gaiolas na fase de berçário em diferentes densidades. Para cada variável medida, letras sobrescritas diferentes na mesma coluna representam diferenças significativas $(\mathrm{P}<0,05)$.

\begin{tabular}{|c|c|c|c|c|}
\hline \multirow{2}{*}{ Variáveis } & \multirow{2}{*}{$\begin{array}{c}\text { Densidades } \\
\text { (ind } / \mathrm{m}^{2} \text { ) }\end{array}$} & \multicolumn{3}{|c|}{ Dias de cultivo } \\
\hline & & 0 & 14 & 28 \\
\hline \multirow{6}{*}{$\begin{array}{l}\text { Concentração } \\
\text { de } \\
\text { clorofila } a\end{array}$} & 100 & $1,89 \pm 0,84^{\mathrm{a}}$ & $3,06 \pm 0,62^{a}$ & $7,15 \pm 2,65^{a}$ \\
\hline & 200 & $1,89 \pm 0,84^{\mathrm{a}}$ & $3,42 \pm 1,02^{\mathrm{a}}$ & $8,30 \pm 0,95^{\mathrm{a}}$ \\
\hline & 300 & $1,89 \pm 0,84^{\mathrm{a}}$ & $2,68 \pm 0,14^{\mathrm{a}}$ & $9,74 \pm 1,10^{\mathrm{a}}$ \\
\hline & 400 & $1,89 \pm 0,84^{\mathrm{a}}$ & $3,03 \pm 0,52^{\mathrm{a}}$ & $7,74 \pm 1,45^{\mathrm{a}}$ \\
\hline & 500 & $1,89 \pm 0,84^{\mathrm{a}}$ & $3,25 \pm 0,32^{\mathrm{a}}$ & $7,95 \pm 1,36^{\mathrm{a}}$ \\
\hline & 100 & $4,81 \pm 4,65^{\mathrm{a}}$ & $4,65 \pm 3,60^{a b}$ & $4,59 \pm 3,82^{\mathrm{a}}$ \\
\hline \multirow{4}{*}{$\begin{array}{l}\text { Densidade de } \\
\text { diatomáceas } \\
\text { cêntricas }\end{array}$} & 200 & $4,81 \pm 4,65^{\mathrm{a}}$ & $7,34 \pm 5,38^{\mathrm{a}}$ & $3,95 \pm 4,33^{\mathrm{a}}$ \\
\hline & 300 & $4,81 \pm 4,65^{\mathrm{a}}$ & $3,26 \pm 2,43^{b c}$ & $4,40 \pm 4,01^{\mathrm{a}}$ \\
\hline & 400 & $4,81 \pm 4,65^{\mathrm{a}}$ & $4,66 \pm 3,92^{b}$ & $2,41 \pm 2,31^{\mathrm{a}}$ \\
\hline & 500 & $4,81 \pm 4,65^{\mathrm{a}}$ & $2,09 \pm 1,75^{\mathrm{c}}$ & $3,61 \pm 2,95^{\mathrm{a}}$ \\
\hline \multirow{5}{*}{$\begin{array}{l}\text { Densidade de } \\
\text { diatomáceas } \\
\text { penadas } \\
\text { grandes }\end{array}$} & 100 & $5,49 \pm 1,88^{a}$ & $5,15 \pm 2,30^{c}$ & $36,09 \pm 5,72^{a}$ \\
\hline & 200 & $5,49 \pm 1,88^{a}$ & $5,56 \pm 1,68^{c}$ & $34,65 \pm 16,05^{a}$ \\
\hline & 300 & $5,49 \pm 1,88^{a}$ & $5,79 \pm 1,09^{b c}$ & $34,06 \pm 5,35^{\mathrm{a}}$ \\
\hline & 400 & $5,49 \pm 1,88^{a}$ & $8,15 \pm 1,88^{\mathrm{b}}$ & $29,32 \pm 6,87^{\mathrm{a}}$ \\
\hline & 500 & $5,49 \pm 1,88^{a}$ & $12,92 \pm 2,53^{a}$ & $31,27 \pm 8,88^{a}$ \\
\hline \multirow{5}{*}{$\begin{array}{l}\text { Densidade de } \\
\text { diatomáceas } \\
\text { penadas } \\
\text { pequenas }\end{array}$} & 100 & $35,54 \pm 7,36^{\mathrm{a}}$ & $28,43 \pm 12,62^{a}$ & $46,04 \pm 13,75^{b}$ \\
\hline & 200 & $35,54 \pm 7,36^{a}$ & $38,81 \pm 10,89^{a}$ & $44,34 \pm 10,40^{b}$ \\
\hline & 300 & $35,54 \pm 7,36^{\mathrm{a}}$ & $26,03 \pm 7,02^{a}$ & $47,05 \pm 7,56^{b}$ \\
\hline & 400 & $35,54 \pm 7,36^{\mathrm{a}}$ & $23,32 \pm 8,31^{a}$ & $39,60 \pm 13,07^{b}$ \\
\hline & 500 & $35,54 \pm 7,36^{\mathrm{a}}$ & $35,50 \pm 21,03^{a}$ & $68,71 \pm 18,34^{a}$ \\
\hline
\end{tabular}


de diatomáceas cêntricas e penadas obtidas naquele estudo tenham sido semelhantes às encontradas no presente, sendo respectivamente, em torno de $6 \times 10^{3}$ células $\mathrm{cm}^{-2}$ e $90 \times 10^{3}$ células $\mathrm{cm}^{-2}$ no estudo anterior e $4 \times 10^{3}$ células $\mathrm{cm}^{-2}$ e $80 \times 10^{3}$ células $\mathrm{cm}^{-2}$ no presente estudo. A diferença nas concentrações finais de clorofila $a$ entre os dois estudos pode estar relacionada a uma possível diferença nas densidades de cianobactérias presentes nos substratos artificiais de ambos os estudos, o que influenciaria nos valores de concentração de clorofila $a$. Outro fator que pode ter contribuído para que fossem observadas diferenças entre as concentrações de clorofila $a$ dos dois estudos, pode ser a possível diferença entre o volume celular das microalgas deste estudo em relação ao estudo anterior.

Observou-se, neste estudo, uma preferência dos camarões pelo consumo de diatomáceas cêntricas, visto que não houve um aumento populacional destas no decorrer do cultivo, ao contrário do que se observou para diatomáceas penadas grandes e diatomáceas penadas pequenas. BORGES et al. (2004) afirmaram que $\boldsymbol{F}$. paulensis tem preferência pela ingestão de diatomáceas penadas maiores que $25 \mu \mathrm{m}$ de comprimento, mas nada se pôde afirmar em relação a diatomáceas cêntricas, uma vez que as condições ambientais daquele estudo não foram favoráveis ao crescimento de diatomáceas cêntricas. É possível que a preferência por diatomáceas cêntricas sugerida, neste estudo, justifique-se pela maior relação volume/ área destas células, o que significa um maior conteúdo intracelular.

Ao final do período experimental, a análise estatística mostrou que a taxa de sobrevivência foi significativamente menor na densidade de $500 \mathrm{PL} / \mathrm{m}^{2}$ (Tabela 3), embora esta não tenha sido significativamente diferente da sobrevivência nas densidades de 200, 300 e 400PL m$~^{-2}$. O tratamento com $100 \mathrm{PL} / \mathrm{m}^{2}$ resultou no maior peso final, enquanto o tratamento com 500PL m${ }^{-2}$ apresentou o menor peso médio, embora não tenha ocorrido diferença significativa entre este e o tratamento com 300PL m${ }^{-2}$ (Tabela 3). A sobrevivência e o crescimento dos camarões diminuíram na medida em que a densidade de estocagem aumentou, o que confirma a existência de uma relação inversamente proporcional entre densidade de estocagem e as taxas de sobrevivência e crescimento. O crescimento dos camarões pode ser considerado satisfatório quando comparados com os resultados de BALLESTER et al. (2003) e LOMBARDI et al. (2003). Estes dois estudos também cultivaram $\boldsymbol{F}$. paulensis em gaiolas, sendo utilizadas $\mathrm{PL}$ com a mesma idade inicial $\left(\mathrm{PL}_{25}\right)$ e períodos de cultivo semelhantes ao do presente estudo (30 e 37 dias, respectivamente). As faixas de peso final obtida nos estudos de BALLESTER et al. (2003), LOMBARDI et al. (2003), bem como no presente estudo, foram respectivamente, $0,65-0,72 \mathrm{~g}, 0,16-0,41 \mathrm{~g}$ e $0,61-1,12 \mathrm{~g}$, e as sobrevivências foram de $91-95 \%, 79-83 \%$ e $61-93 \%$. Vale ressaltar que a densidade de estocagem do primeiro estudo foi de $300 \mathrm{PL} \mathrm{m}^{-2}$, entre 500 e $2000 \mathrm{PL} \mathrm{m}^{-2}$ no segundo estudo e entre 100 e $500 \mathrm{PL} / \mathrm{m}^{2}$ no presente experimento. Em outros estudos com cultivo de camarões em gaiolas na fase de berçário, NATARAJAN et al. (1986) cultivaram Fenneropenaeus indicus na densidade de 100PL m $\mathrm{m}^{-2}$, obtendo sobrevivência de $83 \%$ e peso médio final de $2,5 \mathrm{~g}$. WALFORD \& LAM (1987) testaram diferentes densidades no cultivo de $\boldsymbol{F}$. indicus em gaiolas em berçário e obtiveram sobrevivência de $83 \%$ e peso médio final de $0,48 \mathrm{~g}$ para densidades de até $1700 \mathrm{PL} \mathrm{m} \mathrm{m}^{-2}$.

IWAI (1978) classificou F. paulensis como uma espécie sedentária, caracterizada por não formar cardumes, viver em fundos de areia, areia lodosa ou cascalho, apresentar atividade predominantemente noturna, e, principalmente, por se enterrar no sedimento. Apesar disso, os resultados do presente trabalho demonstraram que $\boldsymbol{F}$. paulensis, embora não estivesse em contato com o sedimento, apresentou sobrevivência e crescimento satisfatórios. D'INCAO (1984) determinou as curvas de crescimento para machos e fêmeas de F. paulensis no ambiente natural, sendo que estes demorariam, respectivamente, 60 e 53 dias para, a partir de $0,014 \mathrm{~g}$, atingirem $0,72 \mathrm{~g}$, peso que, nas condições deste estudo, os camarões atingiram em aproximadamente 35 dias utilizando-se a densidade de 400PL m ${ }^{-2}$, o que confirma o melhor crescimento dos camarões quando há disponibilidade de alimento e proteção contra predadores.

Apesar disso, foi observado que o desempenho dos camarões neste estudo pode ter sido limitado pelas condições ambientais prevalentes

Tabela 3 - Valores médios ( \pm DP) de sobrevivência (\%) e peso final (g) de Farfantepenaeus paulensis criado em gaiolas na fase de berçário em diferentes densidades após 35 dias de cultivo. Letras sobrescritas diferentes na mesma coluna representam diferenças significativas $(\mathrm{p}<0,05)$.

\begin{tabular}{ccc}
\hline $\begin{array}{l}\text { Densidade de } \\
\text { estocagem (ind.m }\end{array}$ & Sobrevivência & Peso \\
\hline 100 & $92,9 \pm 10,8^{\mathrm{a}}$ & $1,12 \pm 0,57^{\mathrm{a}}$ \\
200 & $86,5 \pm 14,8^{\mathrm{ab}}$ & $0,80 \pm 0,32^{\mathrm{b}}$ \\
300 & $85,8 \pm 7,4^{\mathrm{ab}}$ & $0,66 \pm 0,27^{\mathrm{cd}}$ \\
400 & $81,1 \pm 5,5^{\mathrm{ab}}$ & $0,72 \pm 0,32^{\mathrm{bc}}$ \\
500 & $60,9 \pm 10,4^{\mathrm{b}}$ & $0,61 \pm 0,39^{\mathrm{d}}$ \\
\hline
\end{tabular}


durante o período experimental, tais como a baixa salinidade e a baixa concentração de oxigênio dissolvido. Desse modo, pode-se supor que, em condições ambientais mais adequadas, o desempenho desses animais poderia ter sido superior e, possivelmente, poderiam ter sido adotadas densidades de estocagem mais altas.

\section{CONCLUSÃO}

Nas condições do presente estudo, a sobrevivência e o crescimento de $\boldsymbol{F}$. paulensis cultivados em gaiolas na fase de berçário podem ser considerados satisfatórios até $400 \mathrm{PL} \mathrm{m} \mathrm{m}^{-2}$, sendo esta a densidade máxima recomendada para o cultivo em berçários em gaiolas.

Pode-se afirmar também que o biofilme formado nos substratos artificiais é composto em sua maior parte por diatomáceas penadas menores que $50 \mu \mathrm{m}$, seguida pelas diatomáceas penadas maiores ou iguais a $50 \mu \mathrm{m}$ e diatomáceas cêntricas, sendo que $\boldsymbol{F}$. paulensis apresentou uma preferência de consumo deste último grupo de diatomácea. São necessários mais estudos para se verificar se o consumo de diatomáceas cêntricas beneficia de alguma forma a sobrevivência e o crescimento de $\boldsymbol{F}$. paulensis cultivados em gaiola.

\section{AGRADECIMENTOS}

A.L. Preto e T.L. Pissetti agradecem ao CNPq pelas bolsas de mestrado. R.O. Cavalli e P.C. Abreu são bolsistas de produtividade do $\mathrm{CNPq}$.

\section{REFERÊNCIAS}

ABREU, P.C. et al. New perspectives in the use of microorganisms in shrimp culture: food source, water quality and diseases control. Anais do Aqüicultura Brasil'98, Recife, PE, v.2, p.703-709, 1998.

BALLESTER, E.C. et al. Influência do biofilme no crescimento de juvenis do camarão-rosa Farfantepenaeus paulensis cultivados em sistemas de berçário. Atlântica, Rio Grande, v.25, n.2, 2003.

BARDACH, J.E. et al. Aquaculture - the farming and husbandry of freshwater and marine organisms. New York: John Wiley \& Sons, 1972. 868p.

BORGES, L. et al. Análise do consumo alimentar preferencial de juvenis do camarão-rosa Farfantepenaeus paulensis sobre o biofilme em panagens de cultivo. In: CONGRESSO DA SOCIEDADE BRASILEIRA DE AQÜICULTURA E BIOLOGIA AQUÁTICA, 2004, Vitória, ES. Anais... Vitória: Sociedade Brasileira de Aqüicultura e Biologia Aquática, 2004. 464p. p. 80 .
BOYD, C.E. Water quality in warm water fish ponds. Alabama: Auburn University, 1990. 366p.

CAVALli, R.O.; WASIELESKY, W.J. Production of Farfantepenaeus paulensis as bait shrimp in cages: the influence of stocking density. In: THE ANNUAL MEETING OF THE WORLD AQUACUlTURE SOCIETY, 2003, Salvador. Book of abstracts... Salvador: World Aquaculture Society, 2004. 904p. p.164.

CORLETO, F. et al. Crescimento de pós-larvas de Penaeus paulensis, Pérez-Farfante, 1967 em diferentes salinidades. In: ENCONTRO RIO-GRANDENSE DE TÉCNICOS EM AQUiCUlTURA, 4., 1993, Porto Alegre, RS. Anais... Porto Alegre, RS: Universidade Federal do Rio Grande do Sul, 1993. p. 13-23.

D'InCAO, F. Estudo sobre o crescimento de Penaeus (Farfantepenaeus) paulensis Pérez-Farfante, 1967 da Lagoa dos Patos, RS, Brasil. Atlântica, Rio Grande, v.1, p.73-84, 1984.

D'INCAO, F. Taxonomia, padrões populacionais e ecológicos dos dendrobranchiata (Crustacea: Decapoda) do Brasil de do Atlântico ocidental. 1995. 365f. Tese (Doutorado em Ciências na área de Zoologia) Curso de Pós-graduação em Ciências, Universidade Federal do Paraná.

FAO, 2002. World review of fisheries and aquaculture. Capturado em 31 ago. 2004. On line. Disponível no site http://www.fao.org.

HENNIG, O.L.; ANDREATTA, E.R. Effect of temperature in an intensive nursery system for Peaneus paulensis (PérezFarfante, 1967). Aquaculture, v.164, p.167-172, 1998.

IBAMA (Instituto Brasileiro do Meio-Ambiente e dos Recursos Naturais Renováveis) Portaria no $145-\mathrm{N}$, de 29 de outubro de 1998.

IWAI, M. Desenvolvimento larval e pós-larval de Penaeus (Melicertus) paulensis Pérez-Farfante, 1967 (Crustacea, Decapoda) e o ciclo de vida dos camarões do gênero Penaeus da região centro-sul do Brasil. 1978. 137f. Tese (Doutorado em Ciências na area de Zoologia) Curso de Pós-graduação em Ciências, Universidade de São Paulo.

JENSEN, L.V. et al. Growth and survival of pink shrimp Farfantepenaeus paulensis postlarvae in cages and pen enclosures. Scientia Agrícola, v.61, p.332-335, 2004.

LOMBARDI, J.V. et al. Rearing the brazilian pink shrimp Farfantepenaeus paulensis in floating cages: nursery phase. In: THE ANNUAL MEETINGS OF THE WORLD AQUACUlTURE SOCIETY, 2003, Salvador, Brazil. Book of Abstracts... Salvador, Brazil: World Aquaculture Society, 2003. V.1, 480p. p.426.

MARCHIORI, M.A. Guia ilustrado de maturação e larvicultura do camarão-rosa Penaeus paulensis Pérez-Farfante, 1967. Rio Grande, RS: FURG, 1996. 79p.

NATARAJAN, M. V. et al. Cage rearing of early juveniles of the White prawn Penaeus indicus (H. Milne Edwards) for 
stocking in grow out net pens. Journal of Marine Biology Association, India, v.29, n.2, p.387-390, 1986.

NUNES, A.J.P. Camarões marinhos: engenharia e logística operacional de berçários intensivos. Panorama da Aqüicultura, v.12, n.69, p.25-37, 2002

OSTRENSKY, A.; PILCHOWSKY, R.W. Cultivos experimentais de camarões marinhos em tanques-rede no litoral paranaense. In: SIMPÓSIO BRASILEIRO DE AQÜICULTURA, 12., 2002, Goiânia. Anais... Goiânia: Associação Brasileira de Aquicultura, 2002. p. 256

OSTRENSKY, A.; WASIELESKY, W.J. Acute toxicity of ammonia to various life stages of São Paulo shrimp, Penaeus paulensis Pérez-Farfante, 1967. Aquaculture, v.132, p.339347, 1995.

PAQUOTTE, P. et al. Intensive culture of shrimp Penaeus vannamei in cages: zootechnical, economic and environmental aspects. Aquaculture, v.164, p.151-166, 1998.

POERSCH, L.H.; MARCHIORI, M.A. Efeito do oxigênio no camarão rosa Penaeus paulensis Pérez-Farfante, 1967. In: ENCONTRO NACIONAL DE AQUICULTURA (Simpósio Brasileiro de Aquicultura, 7., 1992 - Encontro Brasileiro de Patologistas de Organismos Aquáticos, 2., 1992), Peruíbe, SP. Anais... Peruíbe: Associação Brasileira de Aqüicultura e Associação Brasileira de Patologistas de Organismos Aquáticos, 1992. p. 115

ROCHA, I. et al. A carcinicultura brasileira em 2003. Revista da ABCC, Ano 6, n.1, p.30, 2004

RODRIGUEZ, E.M. et al. Nursery rearing of Penaeus monodon (Fabricius) using suspended (hapa) net enclosures installed in a pond. Aquaculture, Amsterdam, v.112, p.107-111, 1993.

SAMARANAYAKE, R.A.D.B. Pen culture trials with the tiger prawn, Penaeus monodon in Chilaw Laggon, Sri Lanka The Second Asian Fisheries Forum, Manila, Philippines, 991p. p. 107-108, 1990

SANTOS, M.H.S. Alimentação do camarão-rosa Farfantepenaeus paulensis (Pérez-Farfante, 1967) (Decapoda - Penaeidae) cultivado. 2003. 229f. Tese (Doutorado em Oceanografia Biológica) - Curso de Pósgraduação em Oceanografia Biológica, Fundação Universidade Federal do Rio Grande.

SEIFFERT, W. et al. Cultivo de juvenis de $L$. vannamei em viveiros berçários traz flexibilidade ao produtor. Panorama da Aqüicultura, v.13, n.75, p.45-51, 2003

SINGH, H.; MATTAI, S. Preliminary observations on the growth and survival of tiger prawn (Penaeus monodon Fabricius) postlarvae in pen-nurseries. National Symposium on Research and Development in Marine Fisheries, Mandapam Camp, India, v.44, n.2, p.359-363, 1990.

SOARES, R.B. Influência da temperatura na sobrevivência de pós-larvas do camarão-rosa Penaeus paulensis em laboratório. 1996. 36f. Monografia (Curso de Graduação em Oceanologia) - Fundação Universidade Federal do Rio Grande.

SOUZA FILHO, J. et al. Custo de produção do camarão marinho. Florianópolis: IPEA (Instituto de Planejamento e Economia Agrícola) e EPAGRI (Empresa de Pesquisa Agropecuária e Extensão Agrícola), 2002. 24p. (Cadernos de indicadores agrícolas, 1).

SPECK, R.C. et al. Efeitos de diferentes densidades de estocagem sobre o crescimento e a sobrevivência de póslarvas de Penaeus paulensis (Pérez-Farfante, 1967) em sistema de berçário. In: SIMPÓSIO BRASILEIRO SOBRE CULTivo DE CAMARÃO, 4., 1993, João Pessoa, PB. Anais... João Pessoa: MCR Aquacultura, 1993. p.369383.

STONER, A.W.; ZIMMERMAN, R. Food pathways associated with penaeid shrimps in a mangrove-fringed esturary. Fishery Bulletin, v.86, n.3, p.543-551, 1988 .

STURMER, L.N. et al. Intensification of penaeid nursery systems. In: FAST, A.W.; LESTER, L.G. (eds). Culture of Marine Shrimp: principles and practices. Amsterdam: Elsevier Scientific, 1992. p.321-344.

THOMPSON, F.L. et al. Importance of biofilm for water quality and nourishment in intensive shrimp culture. Aquaculture, v.203, p.263-278, 2002

TSUZUKY, M.Y. et al. Effect of salinity on survivor, growth, and oxygen consumption of the pink shrimp Farfantepenaeus paulensis (Pérez-Farfante, 1967). Journal of Shellfish Research, v.22, n.2, p.555-559, 2003.

UNESCO. Chemical methods for use in marine environmental monitoring. Paris: Intergovernmental Oceanographic Commission, 1983. 53p. (Manual and Guides, 12).

WALFORD, J.; LAM, T.J. Floating hatchery and net cage culture of Johore, Singapore. Aquaculture, v.22, p.11-32, 1987.

WASIELESKY, W.J. Cultivo de juvenis do camarãorosa Farfantepenaeus paulensis (Decapoda, Penaeidae) no estuário da Lagoa dos Patos: Efeitos de parâmetros ambientais e manejo de cultivo. 2000. 199f. Tese (Doutorado em Oceanografia Biológica) Rio Grande, Fundação Universidade Federal do Rio Grande.

WASIELESKY, W.J. et al. Crescimento do camarão-rosa Penaeus paulensis (Crustacea: Decapoda) cultivado em gaiolas e cercados, no estuário da Lagoa dos Patos. In: ENCONTRO RIO-GRANDENSE DE TÉCNICOS EM AQUACULTURA, 6. E ENCONTRO SULBRASILEIRO DE AQUACULTURA, 3., 1995, Ibirubá, RS. Anais... Ibirubá, RS: UFRGS, 1995. 155p. p.14-25 